\title{
Expériences rapportées par des psychologues scolaires québécois au primaire et au secondaire sur leurs rôles pour lutter contre l'intimidation à caractère homophobe Reported experiences of Quebec school psychologists in elementary and secondary schools on their roles in countering homophobic bullying
}

\author{
René-Marc Lavigne
}

Volume 6, novembre 2019

URI : https://id.erudit.org/iderudit/1066578ar

DOI : https://doi.org/10.7202/1066578ar

Aller au sommaire du numéro

Éditeur(s)

Université Laurentienne

ISSN

1920-6275 (imprimé)

1929-8544 (numérique)

Découvrir la revue

Citer cet article

Lavigne, R.-M. (2019). Expériences rapportées par des psychologues scolaires québécois au primaire et au secondaire sur leurs rôles pour lutter contre l'intimidation à caractère homophobe. Enfance en difficulté, 6, 37-64. https://doi.org/10.7202/1066578ar

\section{Résumé de l'article}

L'intimidation à caractère homophobe (ICH), une forme d'intimidation qui vise, entre autres, les élèves de minorités sexuelles et de genre, entraine des conséquences psychosociales et scolaires chez les élèves. Les psychologues scolaires, experts en santé mentale, peuvent intervenir lors de situations d'intimidation. Alors que plusieurs études ont évalué les actes homophobes et leurs effets sur les élèves, peu se sont intéressées aux rôles des psychologues scolaires relativement à l'ICH. À l'aide d'entretiens individuels structurés, la présente étude explore les expériences de 30 psychologues scolaires ( 15 hommes, 15 femmes) quant à leurs rôles pour lutter contre l'ICH. Les résultats montrent que la consultation est centrale dans les stratégies conçues par des psychologues pour contrer ce problème. Par exemple, plusieurs participants disent manquer d'expérience professionnelle et de formation concernant l'ICH puisque ce thème est peu enseigné pendant leur formation universitaire. Ils recommandent à leurs collègues psychologues scolaires de s'impliquer davantage en intervention et en sensibilisation en ce qui concerne l'ICH. 
www.laurentienne.ca

\section{Expériences rapportées par des psychologues scolaires québécois au primaire et au secondaire sur leurs rôles pour lutter contre l'intimidation à caractère homophobe}

René-Marc Lavigne

Université du Québec à Montréal

\section{Introduction}

La notion d'intimidation renvoie à une agression caractérisée par l'intentionnalité, la répétition et un déséquilibre du pouvoir (Espelage et Swearer, 2003; Olweus, 1993; Vaillancourt, Hymel et McDougall, 2013). Au moins une adolescente ou un adolescent canadien sur trois est victime d'intimidation (Gouvernement du Canada, 2017). Le problème de l'intimidation dans les écoles entraine diverses conséquences chez les élèves sur les plans psychologique, physique, social et scolaire (Hong et Espelage, 2012; Swearer et Hymel, 2015). Lintimidation peut se manifester par des formes indirectes telles la cyberintimidation ou la propagation de fausses rumeurs, ou des formes directes ou agressives comme les bousculades ou les moqueries (Bégin, 2011; Dehue, Bolman et Vollink, 2008; Siyahhan, Aricak et Cayirdak-Acar, 2012).

Afin de contrer ce problème, l'Assemblée nationale du Québec a adopté en 2012 la Loi visant à prévenir et à combattre l'intimidation et la violence à l'école. Cette loi «prévoit l'obligation, tant pour les établissements d'enseignement publics que pour les établissements d'enseignement privés, d'adopter et de mettre en œuvre un plan de lutte 
contre l'intimidation et la violence " (Gouvernement du Québec, 2012, p. 2). Chaque plan doit prévoir des mesures de prévention, notamment:

[...] préciser les actions qui doivent être prises [...] ainsi que les mesures de soutien ou d'encadrement alors offertes, déterminer les sanctions disciplinaires applicables dans un tel cas et spécifier le suivi qui doit être donné à tout signalement et à toute plainte concernant un acte d'intimidation. (p. 2)

D'autres provinces canadiennes ont adopté des lois semblables dans le but de prévenir l'intimidation et d'intervenir pour la contrer. En 2012, l'Ontario a adopté une loi concernant tout acte d'intimidation et de cyberintimidation qui a une incidence sur le climat d'apprentissage de l'école. Cette loi comprend les droits et les responsabilités des enseignants, des écoles, des conseils scolaires, des autres employés des conseils scolaires et des ministères en matière de prévention ou d'intervention lors de cas d'intimidation (Gouvernement de l'Ontario, 2019). De la même façon, le Code de vie des élèves du Nouveau-Brunswick sert de guide aux écoles en énonçant les comportements souhaités des élèves tels l'empathie, le respect des différences et la dénonciation de l'intimidation (Gouvernement du Nouveau-Brunswick, 2019).

Bien que ces lois prévoient des mesures de lutte contre toute forme d'intimidation à l'école, plus de recherches scientifiques sont requises pour soutenir les élèves provenant des minorités sexuelles et de genre (ÉMSG), soit un groupe particulièrement à risque d'être victime d'intimidation (Espelage, 2016). Les ÉMSG sont de jeunes personnes qui s'identifient comme lesbiennes, gaies, bisexuelles, transsexuelles, transgenres ou non binaires (Gouvernement du Québec, 2017).

\section{Définition et caractéristiques de l'intimidation à caractère homophobe}

Dans le cadre de la présente étude, l'intimidation à caractère homophobe $(\mathrm{ICH})$, une sous-catégorie d'intimidation, renvoie à toute forme d'intimidation envers un ou des ÉMSG. Il s'agit également de tout langage utilisé qui pourrait être dénigrant envers un élève en raison de son orientation sexuelle réelle ou perçue, ou de son expression de genre qui est non conforme aux stéréotypes traditionnels (Boucher et al., 2013; O’Higgins-Norman, Goldrick et Harrison, 2010). 
Les taux d'ICH sont plus élevés chez les jeunes rapportant une attirance exclusivement envers le même sexe (Boucher et al., 2013). L'enquête menée par Taylor et Peter (2011) au Canada auprès de 3700 élèves dans les écoles secondaires montre que $55 \%$ de ces derniers ont été la cible d'ICH s'étant manifestée par des formes verbales telles que des moqueries et $21 \%$ par des formes physiques comme des bousculades. De plus, $64 \%$ des participants se considèrent en danger à l'école en raison de l'ICH. Les résultats de l'enquête de Chamberland, Émond, Julien, Otis et Ryan (2010) auprès de 2747 élèves d'écoles secondaires au Québec révèlent que la majorité des élèves $(86,5 \%)$ entend souvent, ou à l'occasion, des remarques négatives associées à la diversité sexuelle et de genre comme " t'es gai ", "t'es fif » et " t'es une tapette ». Parmi les répondants ÉMSG de l'enquête, 38,6\% de ces derniers disent avoir été la cible d'actes à caractère homophobe tels que des insultes ou la propagation de rumeurs visant à nuire à la réputation.

Selon Chamberland, Richard et Bernier (2013), il existe des différences dans l'incidence d'actes à caractère homophobe selon le sexe des victimes. En effet, il semblerait que les ÉMSG garçons soient plus souvent victimes de bousculades, de coups et d'objets lancés vers eux $(23,4 \%)$ que les filles $(14,4 \%)$. Les ÉMSG filles seraient quant à elles davantage victimes de cyberintimidation à caractère homophobe $(31,4 \%)$ que les garçons $(14,0 \%)$ et seraient également plus à risque $(21,1 \%)$ que les garçons $(10,8 \%)$ de vivre des avances sexuelles insistantes et des attouchements contre leur gré.

De façon générale, les ÉMSG peuvent vivre des difficultés d'ordre psychologique comme des troubles de l'humeur, des troubles anxieux ou encore avoir une faible estime d'eux-mêmes. Les expériences d'ICH peuvent aussi entrainer des conséquences relatives à la persévérance et à la réussite scolaires (Chamberland et al., 2010). Plusieurs ÉMSG victimes d'ICH changent d'école ou souhaitent le faire en raison d'un manque de sentiment de sécurité dans leur milieu (Chamberland et al., 2010; Saewyc et al., 2007). Ces études illustrent l'ampleur des conséquences psychologiques et scolaires de l'ICH, soulèvent l'importance de s'intéresser au phénomène de l'ICH et justifient la pertinence d'examiner en profondeur ce sujet d'étude. Ainsi, il apparait essentiel de déterminer les actions de lutte des écoles contre l'intimidation et l'homophobie. 


\section{Actions de lutte contre l'intimidation et l'homophobie à l'école}

Les écoles se servent de diverses actions pour contrer l'intimidation, abordées dans leurs plans de lutte, telles que les mesures punitives (les retenues, la perte de privilèges, les appels téléphoniques aux parents ou la suspension d'un élève auteur d'intimidation). Le principe de tolérance zéro, souvent annoncé dans les écoles, vise à communiquer aux élèves le fait que les comportements d'intimidation ne seront pas tolérés par les membres du personnel scolaire (Commission scolaire de la Capitale, 2012). Par ailleurs, la majorité des écoles au Canada se sert de programmes d'apprentissage des habiletés sociales visant à prévenir la violence, l'intimidation ou l'ICH en favorisant l'empathie chez les élèves tout en les amenant à réfléchir sur les effets de leurs comportements (Gouvernement du Canada, 2003). Les enseignants abordent le contenu de ces programmes, soit les habiletés socioémotionnelles, dans le cadre de leurs cours. De plus, ils collaborent avec des membres du personnel scolaire afin de renforcer les comportements souhaités et d'offrir un suivi relatif aux apprentissages.

Des organismes communautaires font de la sensibilisation auprès des élèves pour réduire les préjugés et les comportements violents relatifs à l'homosexualité et à l'hétérosexisme. Par exemple, Planned Parenthood Toronto offre le programme Teens educating and confronting homophobia pouvant être composé de témoignages, d'ateliers et de kiosques d'information (Planned Parenthood Toronto, 2019). Par ailleurs, le Groupe de recherche et d'intervention sociale gaie et lesbienne de Montréal (2018) offre gratuitement aux écoles des moyens éducatifs qui visent à sensibiliser le personnel scolaire à l'homosexualité en leur fournissant des exemples de pratiques pour accompagner leurs démarches. Parmi les membres du personnel dans les écoles, les psychologues scolaires, qui sont spécialistes en santé mentale, peuvent aider à déterminer les actions efficaces pour contrer l'intimidation et collaborer aux initiatives de lutte contre l'intimidation ou l'homophobie dans les écoles (Ordre des psychologues du Québec (OPQ), 2007).

\section{Les rôles des psychologues scolaires}

Selon l'OPQ (2007), les principales tâches des psychologues scolaires sont la prévention, l'intervention auprès des élèves, des parents et des groupes ainsi que l'évaluation, la consultation, la formation, la 
recherche, la gestion et le rôle-conseil comme personne-ressource. Selon Leclerc et Beaumont (2014), les psychologues scolaires québécois détiennent des connaissances et des habiletés essentielles relativement à la lutte contre l'intimidation dans les écoles. Par ailleurs, l'OPQ (2007) souligne l'importance du rôle d'intervention des psychologues scolaires qui : "interviennent dans les situations de violence ou d'intimidation» (p. 14). Toutefois, la majorité du temps des psychologues scolaires québécois est consacrée à compléter des évaluations à visée administrative auprès d'élèves en difficulté soupçonnés de requérir des services spécialisés (Gagné, 2010). Selon l'enquête de Béland et Goupil (2005), tenue auprès de 76 psychologues scolaires au primaire, la majorité des participants rapporte que les élèves en difficulté d'apprentissage représentent plus de $50 \%$ de leur clientèle et que $72 \%$ du temps qu'ils consacrent à ces élèves porte sur l'évaluation. Les psychologues scolaires canadiens désirent cependant passer moins de temps à évaluer et offrir une plus grande variété de services tels que l'intervention et la collaboration auprès d'autres membres du personnel scolaire (Jordan, Hindes et Saklofske, 2009).

L'enquête de Sherer et Nickerson (2010), menée auprès de 213 psychologues scolaires américains au moyen de questionnaires à réponses brèves, s'intéresse à leurs perceptions des interventions réalisées dans les écoles relativement à l'intimidation générale. Entre autres, ces professionnels perçoivent les programmes d'apprentissage des habiletés sociales et le changement des locaux ou des horaires des élèves visés par l'intimidation comme étant des mesures de lutte efficaces. Ils jugent peu efficace la séparation physique entre l'auteur d'intimidation et la victime ainsi que le principe de la tolérance zéro. L'étude américaine de Lund, Blake, Ewing et Banks (2012), menée auprès de psychologues et conseillers dans les écoles ( $n=560)$, montre que la plupart des psychologues scolaires obtiennent de la formation et qu'ils ont accès à une documentation convenable concernant les programmes de prévention de l'intimidation. Toutefois, ces professionnels transmettent peu souvent leurs connaissances aux membres du personnel scolaire concernant les outils, les formations ou les mesures de lutte contre ce problème.

Étant donné que les élèves victimes d'ICH requièrent souvent l'intervention psychologique et que les psychologues scolaires possèdent une longue formation et une expertise en santé mentale, la description de leurs expériences quant à leurs rôles relativement à l'ICH parait indispensable pour améliorer les interventions cliniques dans les écoles. 


\section{Les méthodes de recherche qualitatives et l'intimidation}

Patton, Hong, Patel et Kral (2015) ont réalisé une recension systématique de stratégies de recherche provenant d'études qualitatives sur l'intimidation dans divers pays tels le Royaume-Uni, les États-Unis, le Canada, l'Australie, la Norvège, l'Europe centrale et l'Europe de l'Est. Selon ces auteurs, la recherche qualitative sur l'intimidation générale à l'école a principalement consisté à élaborer et à expliquer les expériences des auteurs d'intimidation, des victimes et des témoins, ainsi que des adultes pertinents (p. ex., les parents, les enseignants, les conseillers scolaires) dans leurs propres mots. Les résultats de l'une de ces études ont permis, entre autres, de comparer les perceptions d'élèves avec celles de membres du personnel scolaire quant à la définition conceptuelle du terme intimidation (Mishna, Scarcello, Pepler et Weiner, 2005). Plus précisément, les perceptions d'enseignants du degré de gravité d'un acte de harcèlement ont été ciblées dans le but de déterminer s'il répondait ou non aux critères d'une définition de l'intimidation.

Bien que des études aient porté sur l'intimidation à l'aide de données qualitatives, peu ont fait état des perceptions du psychologue scolaire, spécialiste en santé mentale qui œuvre auprès des élèves. Selon nos recherches dans plusieurs banques de données, telles que Education Resources Information Center, Google Scholar, PsycInfo et PsycArticles, à l'aide de mots-clés (psychologue scolaire, intimidation, homophobie, identité sexuelle et santé mentale), selon les années de publication 2005 à 2017, il semble n'exister aucune étude qualitative québécoise sur les rôles des psychologues quant au problème de l'ICH. Comme les objectifs de cette étude visent l'exploration des connaissances d'un sujet peu documenté, la méthode qualitative parait plus appropriée pour examiner les perceptions des psychologues scolaires quant à leurs rôles pour lutter contre l'ICH.

\section{Objectifs}

Cette étude a pour but de décrire la façon dont les psychologues scolaires québécois voient leurs rôles par rapport à l'ICH. Elle tente de répondre aux questions suivantes :

a) quels sont les rôles des psychologues scolaires en matière d'ICH?;

b) quelles sont leurs interventions visant à contrer l'ICH auprès des victimes, des auteurs d'intimidation et des témoins?;

c) quelle est la contribution des psychologues scolaires aux actions de lutte contre l'ICH?; 
d) quelles sont les recommandations des psychologues scolaires quant aux moyens pouvant servir à améliorer le climat scolaire relatif à l'ICH?

\section{Méthodologie}

Cette section présente les caractéristiques des participants et leur recrutement dans le cadre de l'étude, les instruments utilisés pour la collecte des données, la validation de ces instruments, le déroulement des entretiens ainsi que le mode d'analyse de données.

\section{Participants}

Le Tableau 1 présente les données sociodémographiques relatives aux psychologues participants. On y retrouve des informations telles que le nombre de participants ( $n=30)$, leur âge $(M=45,7$ ans), le nombre d'écoles qu'ils desservent $(M=2,7)$ ainsi que le nombre de demandes de service en ICH reçues annuellement $(M=1,0)$.

\section{Tableau 1}

Nombre de participants ayant différentes caractéristiques sociodémographiques ( $n$ total $=30$ )

\begin{tabular}{|c|c|}
\hline Caractéristiques & Données \\
\hline Sexe & $\begin{array}{l}\text { Hommes }(n=15) \\
\text { Femmes }(n=15)\end{array}$ \\
\hline Âge & $M=45,7$ ans $(E ́ T=10,1)$ \\
\hline Scolarité & $\begin{array}{l}\text { Maitrise }(n=24) \\
\text { Doctorat }(n=6)\end{array}$ \\
\hline $\begin{array}{l}\text { Spécialisation aux cycles } \\
\text { supérieurs }\end{array}$ & $\begin{array}{l}\text { Psychologie clinique }(n=10) \\
\text { Psychologie scolaire }(n=9) \\
\text { Psychologie clinique avec spécialisation adulte }(n=4) \\
\text { Psychologie du développement }(n=3) \\
\text { Psychologie cognitive béhaviorale }(n=3) \\
\text { Neuropsychologie }(n=1)\end{array}$ \\
\hline Années d'expérience & $M=12,5$ années $(E ́ T=9,2)$ \\
\hline Nombre d'écoles & $M=2,7$ écoles $(E ́ T=2,3)$ \\
\hline Type d'écoles desservies & $\begin{array}{l}\text { Primaire }(n=8) \\
\text { Secondaire }(n=8) \\
\text { Primaire et secondaire }(n=14)\end{array}$ \\
\hline
\end{tabular}




\begin{tabular}{|l|l|}
\hline \multicolumn{2}{|c|}{ Tableau 1 (suite) } \\
\hline Caractéristiques & Données \\
\hline Milieux & $\begin{array}{l}\text { Ruraux }(n=16) \\
\text { Urbains }(n=14)\end{array}$ \\
\hline Travail & $\begin{array}{l}\text { Temps plein }(n=17) \\
\text { Temps partiel }(n=13)\end{array}$ \\
\hline Jours de travail par semaine & $M=4,4$ jours $(E ́ T=0,9)$ \\
\hline $\begin{array}{l}\text { Nombre de demandes de services } \\
\text { liées à l'ICH reçues par année }\end{array}$ & $\begin{array}{l}M=1,0 \text { demande }(E ́ T=1,7) \\
\text { Étendue }=0-5\end{array}$ \\
\hline
\end{tabular}

\section{Mode de recrutement}

Les psychologues scolaires ont été recrutés en personne lors du congrès de l'Association québécoise des psychologues scolaires (AQPS). Le critère d'inclusion était de travailler, depuis au moins un an, à titre de psychologue scolaire dans une école du primaire ou du secondaire, au Québec. Les participants admissibles ont été informés du sujet et de la démarche de recherche, soit un entretien individuel semi-structuré de 60 à 90 minutes environ, par visioconférence ou en personne. Ces derniers ont reçu un formulaire de consentement par courriel qui explique les objectifs, le déroulement et les conditions éthiques. Ils ont retourné le questionnaire original signé par la poste. Le comité d'éthique de la recherche avec des êtres humains de la Faculté des sciences humaines de l'Université du Québec à Montréal a approuvé le projet de recherche.

\section{Instruments de collecte de données}

Cette étude se sert d'un devis méthodologique qualitatif. La fiche de renseignements personnels comporte des questions fermées servant exclusivement à la description de l'échantillon. La grille d'entretien est composée de questions ouvertes et semi-ouvertes permettant de répondre aux questions de recherche.

\section{Fiche de renseignements personnels}

La fiche de renseignements personnels comporte 19 questions fermées et recueille les données sociodémographiques telles que l'âge, le dernier diplôme obtenu en psychologie et le nombre d'écoles desservies. 


\section{Grille d'entretien semi-structurée}

Cet article s'inscrit dans le cadre d'une plus vaste étude ayant comme objectifs principaux d'explorer

a) l'implication des psychologues scolaires dans la lutte contre l'ICH,

b) leurs rôles à cet égard et

c) les actions des équipes-écoles pour lutter contre l'ICH.

La grille d'entretien semi-structurée, composée de 20 questions semiouvertes et ouvertes, dont certaines proposent des sous-questions, a été utilisée comme guide. Quatre questions de la grille d'entretien portant sur les rôles des psychologues scolaires ont été retenues et ont composé les thèmes de recherche de cet article. Ces quatre thèmes découlent des objectifs de l'étude et de la littérature scientifique relatives aux psychologues scolaires et à l'ICH. Il s'agit :

a) des rôles professionnels des psychologues scolaires relatifs à l'ICH;

b) des interventions contre l'ICH auprès des victimes, des auteurs d'intimidation et des témoins;

c) de la contribution des psychologues scolaires aux actions de lutte contre l'ICH; et

d) des recommandations des psychologues concernant les moyens pouvant servir à améliorer le climat scolaire relatif à l'ICH (Chamberland et al., 2010; Lefebvre, 2011).

\section{Validation des instruments de collecte de données}

Cinq experts du domaine de la psychologie en éducation ont reçu une grille d'évaluation qui a permis de valider le contenu et d'assurer la conformité de la fiche de renseignements personnels et de la grille d'entretien. Quelques éléments ont été modifiés en fonction des commentaires des experts afin de clarifier leur sens. Ensuite, trois psychologues scolaires ont participé à un entretien pour la préexpérimentation, ce qui a donné lieu à des reformulations mineures.

\section{Déroulement des entretiens}

Les 30 entretiens, y compris la fiche de renseignements personnels, ont été complétés à distance au moyen du logiciel de téléconférence Skype $(n=23)$ ou en personne, dans l'école du participant $(n=7)$. 


\section{Mode d'analyse}

Les réponses aux questions fermées de la fiche de renseignements personnels ont servi à une analyse quantitative, notamment par des fréquences, des moyennes et des écarts types. En ce qui concerne les questions semi-ouvertes et ouvertes de la grille d'entretien, une analyse qualitative de contenu, basée sur la méthode de Miles et Huberman (2003), a été réalisée en trois principales étapes. D'abord, le contenu audionumérique de chaque entretien a été transcrit intégralement. Le chercheur de cette étude a associé des codes numériques aux extraits de la transcription qui ont émergé de la lecture. Ensuite, la création de grilles de codification a permis d'établir une structure thématique. Enfin, le retour aux étapes précédentes a permis la modification des codes, des thèmes et des grilles de codification, et ce, tout au long de l'analyse. Cette analyse se poursuit par une énumération en termes de fréquence des types de réponses.

Afin d'obtenir un accord interjuges, une doctorante en psychologie ayant signé un formulaire de confidentialité a analysé de façon indépendante le contenu de la transcription. Cette analyse comprend la lecture de l'ensemble des extraits transcrits et leur classement en fonction des grilles de codification préétablies par le chercheur de cette étude au moment de l'analyse qualitative de contenu. Le Kappa de Cohen a servi à la validation de chaque classement de la transcription selon le niveau d'accord interjuges. L'indice minimal pour un classement acceptable est de 0,80. L'accord interjuges obtenu pour chacune des grilles de codification se situe entre 0,83 et $1,00(M=91,53 \% ; E ́ T=6,44)$.

\section{Résultats}

Dans le cadre de cette étude, le terme intervention directe renvoie à la relation d'aide individuelle auprès des élèves, à leur soutien et aux conseils qui leur sont offerts; la consultation est liée à l'offre de conseils ou d'impressions cliniques auprès du personnel scolaire; et l'évaluation est en lien avec l'usage de connaissances cliniques afin d'évaluer l'élève.

\section{Les rôles professionnels des psychologues scolaires relatifs à l'ICH}

Les participants ont décrit leurs rôles relatifs à l'ICH et en quoi ils se distinguent de ceux d'autres professionnels scolaires (voir le résumé au Tableau 2). 


\section{Tableau 2}

Nombre de psychologues scolaires ( $\mathrm{n}$ total $=30$ ) qui décrivent leurs rôles professionnels dans la lutte contre l'ICH

\begin{tabular}{|l|l|c|}
\hline Professionnel & Interventions & $\begin{array}{c}\text { Fréquence } \\
\text { de réponse }\end{array}$ \\
\hline Psychologue scolaire & Intervention directe & 24 \\
& Consultation & 13 \\
& Évaluation & 9 \\
& Aucun rôle & 3 \\
\hline Autres professionnels scolaires & Intervention directe & 13 \\
& Sensibilisation & 3 \\
& Implantation de programmes & 1 \\
\hline
\end{tabular}

Lintervention directe et individuelle auprès des victimes ou des auteurs d'intimidation a souvent lieu dans leur bureau. Cette intervention, axée sur la santé mentale de l'élève, est mentionnée par 24 participants :

Mon rôle se distingue par le fait que j'ai une intervention plus de type curatif, soigner la douleur, ou faire une intervention auprès de l'auteur d'intimidation qui serait d'amener un changement [...] de perception. Pour la victime, mon rôle souvent ça va être l'apprentissage de comportements sociaux pour que la personne évite d'être dans une prise de risque. ${ }^{1}$

Un participant mentionne :

Nous sommes habituellement les professionnels qui assurent le suivi de l'élève lorsqu'il est en détresse et nous dirigeons l'élève vers les ressources nécessaires lorsque le suivi ne peut être assumé pleinement à l'école telle qu'une référence au CSSS ou en pédopsychiatrie pour les cas plus sévères.

Un autre participant précise :

[Les membres du personnel scolaire] font référence au psychologue quand il y a des problèmes sous-jacents, l'anxiété,

1 Le masculin est utilisé pour préserver l'anonymat des participantes et des participants, et ce, sans préjudice à la forme féminine. Lorsqu'il a été jugé nécessaire, le texte transcrit a été corrigé dans le but de répondre aux exigences de la langue française afin d'en faciliter la compréhension. 
justement développée suite à [l'ICH], ou des comportements antisociaux ou agressifs ou impulsifs des auteurs d'intimidation, à ce moment-là ils vont faire appel à nous. Comme on est là mais en deuxième ressource souvent. [...] Quand ça se complique, quand ils n'y arrivent pas, quand ils ont l'impression que l'enfant développe quelque chose au niveau psychologique de plus cristallisé, et que l'intervention ne s'avère pas efficace, soit au niveau de l'agressivité de l'auteur d'intimidation ou au niveau de l'anxiété des fois, ou de la tristesse engendrée par tout ça chez la victime, on dirait qu'ils vont faire appel à moi.

Par ailleurs, plusieurs participants $(n=13)$ perçoivent que la consultation auprès d'autres intervenants scolaires s'inscrit dans leurs fonctions de psychologue contre l'ICH : "Nous agissons à titre de consultants dans l'élaboration de la structure de dénonciations, la gestion des plaintes et le choix des intervenants qui s'en chargent. »Un autre participant rapporte ce qui suit :

Le travail de collaboration que j'ai souvent avec ma collègue, c'est des cas où on n'arrive pas à débloquer, à faire bouger les choses. Elle va me consulter, on va discuter et réfléchir ensemble sur ce qu'on peut faire, mais comme elle a souvent commencé les interventions, c'est elle qui va les poursuivre. On s'entraide, on fait un travail de réflexion ensemble, puis on va essayer de dresser le portrait clinique de la situation, c'est quoi les enjeux, c'est quoi l'impact, qu'est-ce qu'il y a en arrière de ça, on va réfléchir à ça ensemble.

Sept participants mentionnent l'évaluation psychologique ou celle du risque suicidaire de l'élève comme faisant partie de leurs rôles: «Nous faisons principalement de l'évaluation cognitive et affective.» Trois participants disent n'exercer aucun rôle relatif à l'ICH en raison d'un manque de disponibilité.

Les psychologues décrivent en quoi consistent leurs rôles quant à l'ICH, se distinguant ainsi du rôle des autres intervenants du milieu scolaire. Parmi ces derniers se trouve le psychoéducateur, membre d'une profession exclusivement québécoise, qui intervient dans le quotidien des personnes qui éprouvent des difficultés d'adaptation, notamment sur le plan comportemental, et favorise l'action sur le terrain (Ordre des psychoéducateurs et psychoéducatrices du Québec, 2018). Treize 
participants mentionnent le rôle d'intervention directe axée sur le comportement de l'élève qui est exercé par le psychoéducateur ou le technicien en éducation spécialisée : «le psychoéducateur va travailler pour que le comportement cesse. Il va traiter des situations et leur contenu, alors que moi, je suis plus un enquêteur, je vais essayer de comprendre ce qui crée cette situation, ce qui la déclenche. » D'autres $(\mathrm{n}=3)$ soulignent le rôle de sensibilisation de ces intervenants : «La psychoéducatrice et la technicienne en éducation spécialisée se sont jumelées et elles passent dans toutes les classes pour parler de l'intimidation, donner des exemples de ça et parler des mécanismes de dénonciation. » La création de programmes de prévention est également mentionnée $(n=1)$.

\section{Les interventions contre l'ICH auprès des victimes, des auteurs d'intimidation et des témoins}

Les participants ont rapporté leurs interventions contre l'ICH (récapitulées au Tableau 3 ) auprès de victimes, d'auteurs d'intimidation et de témoins.

\section{Les interventions auprès des victimes}

Huit participants semblent offrir une relation d'aide aux victimes, alors que six semblent les inciter à répondre aux auteurs d'intimidation :

J'irais voir au niveau de sa tenue corporelle, comment il se tient, regarder dans les yeux, lui apprendre à dire que c'est inacceptable et «je n'accepte pas que tu me parles de cette façon ». Je ferais peut-être des jeux de rôles où je deviendrais l'auteur d'intimidation pour qu'il puisse s'en sortir dans la situation.

D'autres stratégies d'intervention mentionnées sont l'apprentissage de moyens d'éviter l'auteur d'intimidation, le réaménagement de l'horaire, l'apprentissage des habiletés sociales et le développement d'un réseau de soutien : "S'assurer qu'ils ont un réseau [familial] et on a un groupe LGBT vers lequel on peut leur demander d'aller s'ils le veulent.»

\section{Les interventions auprès des auteurs d'intimidation}

Onze participants fourniraient un soutien aux auteurs d'intimidation dans le but de cerner leurs motifs: "J'essaierais de voir ce qui incite l'auteur d'intimidation à faire cela, quel est le gain, quelle est 
son intention, ce que ça lui rapporte. J'amènerais l'élève à réfléchir sur comment il arrive à se valoriser.» D'autres participants disent qu'ils emploieraient des techniques d'autoréflexion avec les auteurs d'intimidation. Certains participants mentionnent la mise en œuvre de sanctions disciplinaires en réponse à l'ICH et d'autres favorisent l'intervention comportementale : "Je me pencherais plus vers une approche comportementaliste, car, dans le contexte scolaire, la première préoccupation est de rétablir une harmonie où chacun évolue avec le même droit sans aucune contrainte. »

\section{Tableau 3}

Nombre de psychologues scolaires ( $\mathrm{n}$ total $=30$ ) qui interviennent auprès des victimes, des auteurs d'intimidation et des témoins d'ICH

\begin{tabular}{|l|l|c|}
\hline Type d'acteur & Interventions & $\begin{array}{c}\text { Fréquence de } \\
\text { réponse }\end{array}$ \\
\hline Victimes & Relation d'aide sur la gestion des émotions & 8 \\
& Apprendre à répondre à l'auteur & 6 \\
& d'intimidation & 2 \\
& Apprendre à éviter l'auteur d'intimidation & 2 \\
& Réaménager l'horaire & 2 \\
& Travailler les habiletés sociales & 2 \\
& Élargir le réseau de soutien & 12 \\
\hline Auteurs & Aucune réponse & 11 \\
d'intimidation & Cerner les motifs des auteurs d'intimidation & 7 \\
& Utiliser des techniques d'autoréflexion & 6 \\
& Imposer des sanctions disciplinaires & 5 \\
& Intervention comportementale & 9 \\
& Aucune réponse & 8 \\
\hline Témoins & Inciter la dénonciation & 7 \\
& Renforcement positif des comportements & 4 \\
& souhaités & 3 \\
& Explorer le vécu affectif & 15 \\
\hline & Explorer le contenu de l'évènement & 4 \\
& Aucune réponse & 2 \\
\hline
\end{tabular}




\section{Les interventions auprès des témoins}

Huit participants inciteraient les témoins d'ICH à dénoncer les actes. D'autres utiliseraient le renforcement positif, comme l'usage de formulations verbales encourageantes ou un reflet thérapeutique sur l'importance du geste posé lorsque les témoins adoptent des comportements souhaités, par exemple lorsqu'ils dénoncent des actes d'ICH. De plus, l'exploration du vécu affectif ou du contenu de l'évènement est mentionnée à quelques reprises : "Si la victime a vécu un acte violent d'ICH, voir quel impact ça a eu sur le témoin. Est-ce qu'il y a de la culpabilité derrière cet aspect [de ne pas avoir dénoncé l'acte auprès de l'école]? Est-ce qu'ils ont peur d'intervenir? » La moitié des psychologues participants $(n=15)$ ne fournit aucun élément de réponse en ce qui concerne les interventions auprès de témoins. Parmi ces derniers, certains mentionnent qu'ils ne sauraient pas quelle intervention préconiser auprès de témoins.

\section{La contribution des psychologues scolaires aux actions de lutte contre l'ICH}

La présente section (résumée au Tableau 4) décrit les réponses des participants concernant la manière dont les psychologues scolaires peuvent contribuer aux actions de lutte contre l'ICH dans les écoles.

\section{Tableau 4}

Nombre de psychologues scolaires (n total $=30$ ) qui décrivent les moyens qu'ils peuvent prendre pour contribuer aux actions de lutte contre l'ICH

\begin{tabular}{|l|c|}
\hline Moyens pouvant contribuer aux actions & Fréquence de réponse \\
\hline Exercer un rôle de consultation & 12 \\
Offrir de la formation sur l'ICH au personnel scolaire & 7 \\
Participer à des activités de sensibilisation & 4 \\
Évaluer l'efficacité des mesures contre l'ICH dans l'école & 3 \\
Observation directe dans l'école & 1 \\
Aucune réponse & 8 \\
\hline
\end{tabular}

Douze psychologues mentionnent le rôle de consultation ou de fonction-conseil dans l'école, notamment auprès des intervenants, des 
enseignants ou de la direction sur les décisions à prendre concernant les cas d'ICH :

Mon rôle pourrait être plus à un niveau de conseil auprès de la direction sur les actions à prendre suite à une situation d'intimidation, ce qui n'est généralement pas fait. Ils ne me demanderont pas si c'est une bonne idée de donner une suspension interne ou externe à l'auteur d'intimidation, mais ils devraient le faire. Surtout lorsque ce sont des élèves ayant des difficultés d'apprentissage.

Par ailleurs :

[Faire] la promotion de ce qui marche. Souvent, l'équipe-école prône des politiques administratives. Le psychologue scolaire, dans sa compréhension de la dynamique des groupes, peut convaincre l'équipe-école du bienfondé d'une approche plus globale, plutôt que de se replier sur des sanctions disciplinaires.

Sept participants voient le psychologue comme un formateur auprès du personnel. Par exemple, l'un d'eux mentionne :

Il est important pour le psychologue de faire réfléchir, entre autres, les autres intervenants sur le besoin psychologique derrière le comportement d'intimidation. L'idée n'est pas d'abolir les interventions, mais, au contraire, de mieux les cibler. C'est une des choses qui manque présentement et clairement. Le psychologue scolaire est bien placé pour offrir cette formation.

Les autres moyens par lesquels les psychologues scolaires peuvent contribuer à l'efficacité des mesures contre l'ICH sont la participation aux activités de sensibilisation $(n=4)$, l'évaluation de l'efficacité des mesures de prévention pour contrer l'intimidation $(\mathrm{n}=3)$ et l'observation directe d'actes d'intimidation dans l'école $(\mathrm{n}=1)$. Huit participants ne donnent aucune réponse à cette question.

\section{Les recommandations des psychologues scolaires pour lutter contre l'ICH}

Cette section offre un aperçu des réponses relatives aux recommandations pour lutter contre l'ICH auprès des quatre sous-groupes suivants : psychologues scolaires, directions, enseignants et élèves. Le Tableau 5 regroupe ces recommandations. 


\section{Tableau 5}

Nombre de psychologues scolaires ( $n$ total $=30$ ) ayant fait des recommandations sur l'ICH

\begin{tabular}{|c|c|c|}
\hline $\begin{array}{l}\text { Recommandations } \\
\text { s'adressant aux : }\end{array}$ & Thèmes de réponse & $\begin{array}{l}\text { Fréquence } \\
\text { de réponse }\end{array}$ \\
\hline Psychologues & $\begin{array}{l}\text { S'impliquer dans l'intervention } \\
\text { S'impliquer dans la sensibilisation } \\
\text { S'impliquer dans la consultation } \\
\text { Aller chercher de la formation } \\
\text { Aucune réponse }\end{array}$ & $\begin{array}{l}8 \\
8 \\
7 \\
4 \\
9\end{array}$ \\
\hline Directions & $\begin{array}{l}\text { Prioriser les moyens de sensibilisation } \\
\text { Offrir de la formation au personnel } \\
\text { Créer des regroupements multidisciplinaires } \\
\text { Aucune réponse }\end{array}$ & $\begin{array}{l}14 \\
5 \\
4 \\
7\end{array}$ \\
\hline Enseignants & $\begin{array}{l}\text { Dénoncer les actes d'intimidation } \\
\text { Intervenir informellement } \\
\text { Recevoir de la formation } \\
\text { S'impliquer dans la sensibilisation } \\
\text { Intégrer du contenu éducatif dans les cours } \\
\text { Aucune réponse }\end{array}$ & $\begin{array}{c}10 \\
4 \\
3 \\
2 \\
1 \\
12\end{array}$ \\
\hline Élèves & $\begin{array}{l}\text { Adopter un esprit d'ouverture face à la diversité } \\
\text { Dénoncer les actes d'intimidation } \\
\text { Rappel de la présence de sanctions } \\
\text { disciplinaires } \\
\text { Aucune réponse }\end{array}$ & $\begin{array}{c}13 \\
7 \\
3\end{array}$ \\
\hline
\end{tabular}

\section{Psychologues}

Huit participants recommandent à leurs collègues psychologues de s'impliquer davantage dans l'intervention contre l'ICH :

Souvent une rencontre peut être tout à fait déterminante dans la vie d'un jeune. Je sais qu'on est souvent coincé dans notre rôle, mais je pense que parfois même une seule rencontre, une seule occasion pour un jeune de s'ouvrir en contexte clinique, peut faire la différence. Souvent par la suite, on a des nouvelles comme quoi les jeunes cheminent bien et sont heureux. 
Par ailleurs, huit participants mentionnent que les psychologues scolaires devraient sensibiliser les parents et les élèves aux problématiques d'intimidation et d'homophobie $(n=8)$ : «Les parents devraient avoir des petits ateliers, à la fin du primaire ou au secondaire : c'est quoi l'intimidation, quels signes pourrait-on reconnaitre chez nos jeunes quand ils en vivent, qu'est-ce qui pourrait apparaitre comme symptôme.» Les psychologues peuvent également offrir de la consultation :

Les psychologues scolaires, on devrait se préoccuper de l'ensemble de l'école, pas juste regarder les cas d'enfants en difficulté. On a une expertise naturellement de par qui on est, de par notre formation, et je crois que [l'ICH] c'est un domaine qu'on devrait s'approprier. On devrait s'impliquer avec les directions d'école pour mettre en place des programmes.

Six participants n'offrent aucune réponse en raison de leur faible expérience en ce qui concerne ce problème : «Je serais plutôt prêt à entendre d'autres psychologues me dire ce qui est vécu, je suis peutêtre aveugle, mais je n'ai pas l'impression que c'est un problème qui est fréquent dans un petit milieu comme celui où je travaille.»

\section{Directions scolaires}

Quatorze participants recommandent aux directions scolaires de prioriser les moyens de sensibilisation, notamment à visée éducative, auprès des auteurs d'intimidation :

Je souhaiterais que la direction rencontre les auteurs d'intimidation, mais que cette rencontre soit aussi à visée éducative et qu'il y ait des recherches de solutions avec cet auteur d'intimidation. C'est un discours que je donne souvent aux directions. Donc, faites appel aux intervenants externes pour sensibiliser nos élèves auteurs d'intimidation à l'impact de ce qu'ils font.

Les regroupements professionnels multidisciplinaires relatifs aux problèmes d'intimidation, d'homophobie et de transphobie sont mentionnés à quelques reprises $(n=4)$ : «Je verrai ça plus en collaboration, avoir un petit comité formé de professionnels et d'enseignants qui veut réfléchir à ça et mettre les choses en place. Dans un petit groupe, on travaille plus ensemble pour chercher des ressources 
extérieures". La formation destinée au personnel scolaire sur la diversité sexuelle et de genre est également mentionnée : «Comme tous les intervenants n'arrivent pas avec une formation, une sensibilisation ou une connaissance par rapport au développement et à la diversité sexuelle, il faudrait que ça soit intégré aux formations continues parce que le personnel change.»

\section{Enseignants}

Le tiers des participants $(n=10)$ suggère aux enseignants de dénoncer les actes d'ICH lorsqu'ils en sont témoins :

Il faut amener les enseignants à ouvrir l'esprit à leurs élèves par rapport à l'intimidation à caractère homophobe et apprendre à dénoncer ou être plus à l'affut des situations qui se passeraient dans le corridor. Si les enseignants intervenaient tous auprès des victimes et appliquaient le protocole de façon systématique, cela pourrait déjà beaucoup aider.

Quatre participants soulignent l'importance d'offrir, de façon informelle, de l'intervention ponctuelle auprès des élèves : "Ils ont un rôle tellement important et souvent ils le sous-estiment. Ils croient qu'ils vont être rapidement oubliés, mais le fait de prendre quelques minutes avec un élève c'est souvent ce moment qui va changer sa vie positivement." D'autres recommandations sont de recevoir de la formation sur l'intimidation ou la diversité sexuelle et de genre, de s'impliquer dans la sensibilisation et d'intégrer du contenu éducatif sur la diversité dans leurs cours.

\section{Élèves}

Près de la moitié des participants $(n=13)$ suggère aux élèves d'adopter un esprit d'ouverture face à la diversité afin de favoriser un climat de respect dans l'école : «On mérite tous le respect, d'être valorisé, reconnu dans ce qu'on est, on a le droit d'être différent, on peut être noir, blanc, avoir les cheveux roux, blonds, peu importe, être catholique, musulman, on doit se respecter. » Inciter les témoins à dénoncer les actes d'ICH est mentionné à quelques reprises : "Leur insuffler le courage de parler aux bonnes personnes, les intervenants, les professeurs, quitte à se faire accompagner par un ami quand on fait la démarche, puis oser dénoncer les gestes. » Plusieurs participants ne répondent pas à la question sur les recommandations en précisant qu'ils n'ont rien de plus à rajouter à ce qu'ils ont mentionné aux questions précédentes. 


\section{Discussion}

La discussion aborde les thèmes découlant des objectifs de l'étude, soit les rôles professionnels des psychologues scolaires quant à l'ICH, leurs interventions, leurs contributions aux actions des écoles et leurs recommandations pour lutter contre l'ICH. Par la suite, les recommandations cliniques, les pistes de recherche ultérieures, les limites et les apports de cette étude sont exposés.

Les participants de cette étude $(n=30)$ disent faire de l'intervention directe et individuelle contre l'ICH et recommandent à leurs collègues psychologues d'en faire davantage. Toutefois, les participants interviennent peu dans les cas d'ICH. Ils ont en moyenne reçu seulement 1,0 demande de service liée à l'ICH dans la dernière année ( $E ́ T=1,7$; Étendue $=0-5$ ). Ainsi, les psychologues devraient prioriser les actions de prévention contre l'ICH, car les élèves victimes vont peu souvent chercher de l'aide. Par ailleurs, ces professionnels ne précisent pas que les psychologues scolaires devraient ajuster ou modifier des techniques d'intervention précises relativement à l'ICH. Considérant ces résultats ainsi que leur longue formation en intervention auprès des élèves, des parents et des groupes, ainsi qu'en consultation, en formation et en recherche $(\mathrm{OPQ}, 2007)$, les psychologues scolaires semblent qualifiés dans leur rôle d'intervention contre l'ICH dans les écoles. De plus, comme le soulèvent les psychologues ayant participé à l'étude de Lund et al. (2012), les psychologues scolaires devraient privilégier une intervention axée sur les besoins psychologiques des auteurs d'intimidation plutôt qu'une approche punitive telle qu'elle est souvent constatée dans les plans d'intervention contre l'intimidation dans les écoles.

Plusieurs participants disent qu'un de leurs rôles liés à l'ICH est la consultation ou l'offre de conseils auprès du personnel scolaire. En effet, des participants situent leur rôle de consultation au premier rang comme moyen de contribuer à la lutte contre l'ICH dans les écoles. La consultation permet aux psychologues de prodiguer des conseils, de mettre leurs connaissances au service de l'équipe multidisciplinaire et de développer le sentiment de compétence professionnelle des membres de cette équipe. En effet, le sentiment de collaboration professionnelle multidisciplinaire des intervenants scolaires contribue à l'amélioration des mesures de prévention contre l'ICH (O'Brennan, Waasdorp et Bradshaw, 2014). Limplantation de regroupements professionnels multidisciplinaires relatifs à l'ICH pourrait effectivement favoriser la communication, l'entraide et l'interdisciplinarité. En ce sens, les 
psychologues scolaires pourraient jouer un rôle-conseil auprès des membres du personnel scolaire, des familles, des services des centres communautaires ainsi que des autres psychologues. Toutefois, ce travail est-il suffisant pour permettre à l'équipe-école de répondre aux urgences liées à l'ICH, considérant la lourde tâche des psychologues? Près de la moitié des participants ( $43 \%)$ travaille à temps partiel et la moyenne des écoles desservies pour l'ensemble est de 2,7. Lintervention et la consultation au regard de l'ICH s'ajoutent donc à un horaire chargé dans un contexte où le nombre d'écoles desservies limite l'implication dans chacune d'entre elles.

Les psychologues scolaires soulignent une variété de techniques d'intervention auprès des élèves impliqués dans l'ICH, dont la relation d'aide axée sur la gestion des émotions et la détermination des motifs d'ICH auprès des auteurs d'intimidation. Bien que ces interventions correspondent aux besoins des élèves, selon les psychologues, il serait pertinent d'évaluer les effets d'une plus grande période de temps en intervention directe contre l'ICH consacrée aux psychologues scolaires, compte tenu du contexte scolaire qui peut limiter l'exercice de leurs fonctions. Pour ce faire, les psychologues et les directions scolaires devraient se réunir régulièrement pour discuter des avantages ou désavantages d'une telle implication.

En ce qui concerne la contribution des psychologues scolaires aux actions de lutte contre l'ICH, ces derniers disent vouloir occuper un rôle d'évaluation et de validation clinique de l'efficacité des actions de lutte contre l'ICH dans les écoles. Ce rôle permettrait au psychologue scolaire de mettre à profit son expertise en évaluation. Le psychologue pourrait ainsi évaluer le plan de lutte contre l'intimidation établi dans l'école et s'assurer qu'il comprend les mesures d'intervention destinées au soutien auprès des ÉMSG. Par ailleurs, les psychologues ayant participé à cette étude désirent offrir plus de consultation en contexte d'ICH auprès du personnel et de la direction scolaire. Ce résultat correspond aux éléments décrits par Jordan et al. (2009) concernant les rôles souhaités par les psychologues scolaires. Il apparait essentiel que ce professionnel puisse exercer un plus grand éventail de fonctions professionnelles relatives à l'ICH, considérant qu'il détient une expertise en santé mentale des élèves. Cette proposition soutient que les directions scolaires devraient soutenir et encourager le perfectionnement professionnel des psychologues scolaires selon leurs divers champs de compétences.

Il apparait alarmant que plus de la moitié des psychologues participants ne fournisse pas la recommandation aux élèves d'adopter 
un esprit d'ouverture à la diversité sexuelle et de genre. Par ailleurs, les participants n'ont pas recommandé aux psychologues de faire davantage de recherche relative à l'ICH dans les écoles alors qu'ils ont consacré plusieurs années de leur formation universitaire à des tâches de recherche. Des explications de ces résultats peuvent se retrouver dans la littérature scientifique sur les difficultés liées à la mobilisation des connaissances dans le milieu de pratique. Une enquête menée auprès de professionnels détenant des rôles de leadeurship dans onze conseils scolaires au Canada montre que les activités de mobilisation des connaissances dépendent du volontariat de personnes intéressées au sein des écoles, plutôt que de leur intégration au travail quotidien des divers professionnels scolaires (Levin, Cooper, Arjomand et Thompson, 2011). Moins de la moitié des responsables en éducation participant à l'enquête de Levin et al., (2011) a indiqué que des services de recherche existent au sein de leur conseil scolaire. De plus, les obstacles majeurs à l'intégration sont le manque de temps, l'absence de capacité ou de personnes prenant l'initiative et la faible priorité accordée aux activités. Alors que les psychologues ayant participé à notre étude disent vouloir offrir de la consultation relative à l'ICH auprès du personnel scolaire, il semble primordial que ces derniers reçoivent d'abord du perfectionnement professionnel sur la diversité sexuelle et de genre. Pour ce faire, les directions scolaires pourraient intégrer l'utilisation de la recherche aux équipes et aux comités interprofessionnels dans les écoles. De plus, ils pourraient mettre en œuvre des réseaux pour susciter l'apprentissage au sujet de la recherche sur l'ICH au sein des écoles et des conseils scolaires. Comme le soulignent les psychologues participants, les directions scolaires devraient mettre en place plus de mesures de sensibilisation à l'ICH. Ils pourraient aussi intégrer une section ICH au plan de lutte contre l'intimidation dans leur école, ce qui permettrait ainsi aux élèves de consulter un intervenant au sujet de ce problème précis. Pour ce faire, les directions devraient faire la promotion des services psychologiques dans les écoles. Dans le but d'être mieux outillés afin d'offrir un soutien de qualité aux élèves qui fréquentent les écoles, les programmes universitaires en psychologie pourraient offrir des modèles d'intervention reconnus pour lutter contre l'ICH.

\section{Pistes de recherche ultérieures}

D'abord, la Loi visant à prévenir et à combattre l'intimidation et la violence à l'école (Gouvernement du Québec, 2012) ne fait pas mention, de façon explicite, des rôles des psychologues scolaires. De son côté, le 
guide intitulé Cadre de pratique des psychologues scolaires exerçant en milieu scolaire (OPQ, 2007) mentionne le rôle du psychologue lié à l'intimidation. Enfin, une harmonisation entre ces deux documents parait justifiée puisqu'une quantité importante de recherches ont été effectuées au Québec concernant ce problème depuis la publication du guide.

Ensuite, il apparait primordial de mener d'autres recherches quant aux rôles que peuvent jouer les psychologues scolaires afin que leur expertise soit mise à profit dans les écoles. Aussi, dans la logique de prise de distance par rapport au psychologue scolaire, il serait intéressant de concevoir une étude descriptive d'histoires de cas relatées par un psychologue où le sujet n'est pas le psychologue scolaire, mais bien l'école. Une telle étude pourrait s'intéresser, entre autres, aux statistiques sur l'ICH, aux plans de lutte, aux réalités organisationnelles des services d'intervention reliés à l'ICH et à l'impact de ce problème sur les élèves victimes, les auteurs d'intimidation et les témoins. Enfin, dans un contexte sociétal où la diversité sexuelle et de genre est de plus en plus abordée, il serait intéressant et pertinent de mener une étude en parallèle avec celles ayant porté sur l'intimidation liée aux communautés culturelles.

\section{Limites et apports de l'étude}

La présente étude comporte certaines limites. D'abord, plusieurs participants n'ont pas pu répondre à certaines questions. Un échantillon représentatif de répondants de différentes professions aurait permis une vision plus nuancée de la réalité de l'ICH dans les écoles et de cerner davantage les besoins relatifs à l'ICH. Cela aurait aidé à déterminer si les autres intervenants tiennent un discours semblable ou non à celui des psychologues scolaires. Par ailleurs, les participants ont offert plusieurs commentaires sur les rôles généraux des psychologues scolaires, alors qu'aucune question de la grille d'entretien ne portait sur ceux-ci. Il est alors possible qu'ils aient répondu aux questions en fonction de leurs connaissances générales de l'intimidation en milieu scolaire et de l'ICH plutôt qu'en fonction de leur expérience professionnelle. Ces données n'ont pas pu être traitées formellement étant donné qu'elles ne sont pas étroitement liées au sujet de l'étude.

Le principal apport de la présente étude est l'analyse des rôles des psychologues scolaires concernant l'ICH, un thème d'actualité qui est très complexe. Cette étude expose l'importance d'impliquer davantage 
les psychologues scolaires aux actions de lutte contre ce phénomène. Elle précise les rôles que souhaitent se voir attribuer les psychologues scolaires concernant l'ICH dans les écoles et offre des pistes de réflexion tant pour la communauté scientifique que pour les professionnels scolaires. De plus, les recommandations des psychologues contribuent au perfectionnement des plans de lutte contre l'intimidation et aux actions menées par les équipes-écoles pour lutter contre ce problème.

\section{Références}

Bégin, M. (2011). La cyberintimidation: une étude exploratoire sur le point de vue des adolescents. Mémoire de maitrise inédit, Université de Sherbrooke. Repéré à www.collectionscanada.gc.ca/obj/thesescanada/ vol2/002/MR88853.PDF

Béland, K. et Goupil, G. (2005). Pratiques et perceptions des psychologues scolaires face à l'évaluation des élèves en difficulté d'apprentissage au Québec. Canadian Journal of School Psychology, 20(1/2), 44-61. doi: 10.1177/0829573506295707

Boucher, K., Blais, M., Hébert, M., Gervais, J., Banville-Côté, C., Bédard, I.,... l'Équipe de recherche PAJ. (2013). La victimisation homophobe et liée à la non-conformité de genre et l'adaptation scolaire et psychosociale chez les 14-22 ans : résultats d'une enquête québécoise. Recherches \& Éducations, 8(1), 83-98.

Chamberland, L., Émond, G., Julien, D., Otis, J. et Ryan, W. (2010). L’impact de l'homophobie et de la violence homophobe sur la persévérance et la réussite scolaires. Montréal, QC: Université du Québec à Montréal. Repéré à www.frqsc.gouv.qc.ca/documents/11326/535055/ PT_ChamberlandL_annexes+2010_impact+homophobie+vs+PRS/ 0b059488-ad2a-485b-9339-6c5b4188b2c6

Chamberland,L., Richard, G. et Bernier,M. (2013). Les violences homophobes et leurs impacts sur la persévérance scolaire des adolescents au Québec. Recherches \& Éducations, 8, 81-96.

Commission scolaire de la Capitale. (2012). Protocole d'intervention pour contrer l'intimidation et la violence. Repéré à https://cscapitaleecole-desjeunes-du-monde.ca/wp-content/uploads/2014/01/protocoleintimidation-ecole-des-jeunes-du-monde-final.pdf

Dehue, F., Bolman, C. et Vollink, T. (2008). Cyberbullying: Youngsters' experiences and parental perception. CyberPsychology \& Behavior, 11, 217-223. doi: $10.1089 /$ cpb.2007.0008

Espelage, D. L. (2016). Sexual orientation and gender identity in schools: A call for more research in school psychology - No more excuses. Journal of School Psychology, 54, 5-8. doi: 10.1016/j.jsp.2015.11.002 
Espelage, D. L. et Swearer, S. M. (2003). Research on school bullying and victimization: What have we learned and where do we go from here? School Psychology Review, 32, 365-383.

Gagné, R. (2010). Lintimidation entre enfants ne fait pas partie du DSM. Psychologie Québec, 27(2), 22-24.

Gouvernement de l'Ontario. (2019). Projet de loi n ${ }^{0} 13$ : Loi modifiant la Loi sur l'éducation en ce qui a trait à l'intimidation et à d'autres questions. Toronto, ON: Assemblée législative de l'Ontario. Repéré à www.ola.org/ fr/affaires-legislatives/projets-loi/legislature-40/session-1/projet-loi-13

Gouvernement du Canada. (2003). Guide d'implantation des programmes d'habiletés sociales et de résolution de conflits en milieu scolaire. Montréal, QC: Centre international de résolution de conflits et de médication. Repéré à www.preventionscolaire.ca/doc/implantation.pdf

Gouvernement du Canada. (2017). Les statistiques de l'intimidation au Canada. Repéré à www.cihr-irsc.gc.ca/f/45838.html

Gouvernement du Nouveau-Brunswick. (2019). Lignes directrices du code de vie des élèves du Nouveau-Brunswick. Fredericton, N-B: Ministère de l'éducation du Nouveau-Brunswick. Repéré à

https://www2.gnb.ca/content/dam/gnb/Departments/ed/pdf/K12/ policies-politiques/f/703FD.pdf

Gouvernement du Québec. (2012). Loi visant à prévenir l'intimidation et la violence à l'école, projet de loi n $\mathrm{n}^{\circ} 56$ (sanctionné - 15 juin 2012), $2^{\mathrm{e}}$ sess., 39e légis. (QC) Repéré à http://www.education.gouv.qc.ca/contenuscommuns/etablissements-scolaires-publics-et-prives/intimidation-etviolence-a-lecole/projet-de-loi/

Gouvernement du Québec. (2017). Plan d'action gouvernemental de lutte contre l'homophobie et la transphobie. Québec, QC: Bibliothèque et archives nationales du Québec. Repéré à www.justice.gouv.qc.ca/ fileadmin/user_upload/contenu/documents/Fr_francais_/centredoc/ publications/ministere/plans-actions/Plan_action_gouvernemental_ lutte_contre_homophobie_transphobie_2017-2022.pdf

Groupe de recherche et d'intervention sociale gaie et lesbienne-Montréal. (2018). Rapport annuel 2017-2018. Montréal,QC:Gris-Montréal.Repéréà www.gris.ca/app/uploads/2018/09/00_a.Gris-Rapport2017-18-WEB.pdf

Hong, J. S. et Espelage, D. L. (2012). A review of research on bullying and peer victimization in school: An ecological systems analysis. Aggression and Violent Behavior, 17, 311-322. doi: 10.1016/j.avb.2012.03.003

Jordan, J. J., Hindes, Y. L. et Saklofske, D. H. (2009). School psychology in Canada: A survey of roles and functions, challenges and aspirations. Canadian Journal of School Psychology, 24, 245-264. doi: 10.1177/0829573509338614

Leclerc, D. et Beaumont, C. (2014). Violence et intimidation à l'école: caractéristiques et défis pour l'intervention. Revue québécoise de psychologie, 35(2), 1-4. 
Lefebvre, M. (2011). Exploration et réflexion sur le rôle du psychologue scolaire au Québec. Essai de doctorat inédit, Université du Québec à Trois-Rivières. Repéré à http://depot-e.uqtr.ca/2697/1/030295535.pdf

Levin, D., Cooper, A., Arjomand, S. et Thompson, K. (2011). L'utilisation de la recherche et son incidence dans les écoles secondaires: une étude examinant la mobilisation des connaissances en éducation. Toronto, ON: Association canadienne d'éducation. Repéré à https://www.edcan.ca/wpcontent/uploads/ace-2011-lutilization-de-la-recherche.pdf

Lund, E. M., Blake, J. J., Ewing, H. K. et Banks, C. S. (2012). School counselors' and school psychologists' bullying prevention and intervention strategies: A look into real-world practices. Journal of School Violence, 11, 246-265. doi: 10.1080/15388220.2012.682005

Miles, M. B. et Huberman, A. M. (2003). Analyse des données qualitatives ( $2^{\mathrm{e}}$ éd.). Paris, France: Sage.

Mishna, F., Scarcello, I., Pepler, D. et Wiener, J. (2005). Teachers' understanding of bullying. Canadian Journal of Education, 28, 718-738. doi: 10.2307/4126452

O’Brennan, L. M., Waasdorp, T. E. et Bradshaw, C. P. (2014). Strengthening bullying prevention through school staff connectedness. Journal of Educational Psychology, 106(3), 870-880. doi: 10.1037/a0035957

O’Higgins-Norman, J., Goldrick, M. et Harrison, K. (2010). Addressing homophobic bullying in second-level schools. Dublin, Irlande: The Equality Authority.

Olweus, D. (1993). Bullying at school: What we know and what we can do. Cambridge, MA: Blackwell. doi: 10.1002/pits.10114

Ordre des psychoéducateurs et psychoéducatrices du Québec. (2018). Le psychoéducateur. Repéré à www.ordrepsed.qc.ca/fr/grand-public/lepsychoeducateur/

Ordre des psychologues du Québec. (2007). Cadre de pratique des psychologues en milieu scolaire. Québec, QC: Ordre des psychologues du Québec. Repéré à https://aqps.qc.ca/public/cadrepratique.pdf

Patton, D. U., Hong, J. S., Patel, S. et Kral, M. J. (2015). A systematic review of research strategies used in qualitative studies on school bullying and victimization. Trauma, Violence, \& Abuse, 18(1), 1-14. doi: $10.1177 / 1524838015588502$

Planned Parenthood Toronto. (2019). Teens educating and confronting homophobia-t.e.a.c.h.Toronto, ON:PlannedParenthoodToronto.Repéréà www.ppt.on.ca/services-and-programs/community-programming/teenseducation-and-confronting-homophobia-t-e-a-c-h/

Saewyc, E., Poon, C., Wang, N., Homma, Y., Smith, A. et McCreary Centre Society (2007). Not yet equal: The health of lesbian, gay, \& bisexual youth in BC. Vancouver, C-B: McCreary Centre Society. 
Sherer, Y. C. et Nickerson, A. B. (2010). Anti-bullying practices in American schools: Perspectives of school psychologists. Psychology in the Schools, 47(3), 217-229. doi: 10.1002/pits.20466

Siyahhan, S., Aricak, O. T. et Cayirdak-Acar, N. (2012). The relation between bullying, victimization and adolescents' level of hopelessness. Journal of Adolescence, 35, 1053-1059. doi: 10.1016/j.adolescence.2012.02.011

Swearer, S. M. et Hymel, S. (2015). Understanding the psychology of bullying: Moving toward a social-ecological diathesis-stress model. American Psychologist, 70, 344-353. doi: 10.1037/a0038929

Taylor, C. et Peter, T. (2011). "We are not aliens, we're people, and we have rights." Canadian human rights discourse and high school climate for LGBTQ students. Canadian Review of Sociology, 48, 275-312. doi: 10.1111/j.1755-618X.2011.01266.x

Vaillancourt, T., Hymel, S. et McDougall, P. (2013). The biological underpinnings of peer victimization: Understanding why and how the effects of bullying can last a lifetime. Theory Into Practice, 52, 241-248. doi: 10.1080/00405841.2013.829726

\section{Correspondance :}

René-Marc Lavigne

Centre de santé intégrale St-Denis

6994, rue St-Denis

Montréal, Québec

Canada, H2S 2S4

Tél. : +1 (438) 921-0760

Courriel : lavigne.rene1@gmail.com

\section{Résumé}

Expériences rapportées par des psychologues scolaires québécois au primaire et au secondaire sur leurs rôles pour lutter contre l'intimidation à caractère homophobe. Lintimidation à caractère homophobe (ICH), une forme d'intimidation qui vise, entre autres, les élèves de minorités sexuelles et de genre, entraine des conséquences psychosociales et scolaires chez les élèves. Les psychologues scolaires, experts en santé mentale, peuvent intervenir lors de situations d'intimidation. Alors que plusieurs études ont évalué les actes homophobes et leurs effets sur les élèves, peu se sont intéressées aux rôles des psychologues scolaires relativement à 
l'ICH. À l'aide d'entretiens individuels structurés, la présente étude explore les expériences de 30 psychologues scolaires (15 hommes, 15 femmes) quant à leurs rôles pour lutter contre l'ICH. Les résultats montrent que la consultation est centrale dans les stratégies conçues par des psychologues pour contrer ce problème. Par exemple, plusieurs participants disent manquer d'expérience professionnelle et de formation concernant l'ICH puisque ce thème est peu enseigné pendant leur formation universitaire. Ils recommandent à leurs collègues psychologues scolaires de s'impliquer davantage en intervention et en sensibilisation en ce qui concerne l'ICH.

Mots-clés : psychologue scolaire; rôles; intimidation; homophobie; minorités sexuelles; santé mentale.

\section{Abstract}

Reported experiences of Quebec school psychologists in elementary and secondary schools on their roles in countering homophobic bullying. Homophobic bullying, which can target students of diverse sexual orientations and gender identities, results in psychosocial and educational consequences for students. School psychologists, experts in mental health and child development, can intervene in situations of bullying and violence. While several studies have assessed the nature of homophobic acts and their effects on students, few have examined the roles of school psychologists concerning homophobic bullying. This study explores the self-reported experiences of thirty school psychologists on their roles in countering homophobic bullying. Results show that consultation is a central component of the school psychologist's developed strategies in addressing this problem. Also, participants report that they lack professional experience and training regarding homophobic bullying because this subject is rarely taught during their graduate studies. They recommend that school psychologists become more involved in intervention and awareness promotion in regards to homophobic bullying.

Keywords: school psychologist; roles; bullying; homophobia; sexual minority; mental health. 